\title{
Big Data Research on Keloids in Atopic Dermatitis Patients with Allergic Comorbidities: Increased Prevalence of Keloids in Atopic Dermatitis
}

Hyo-eun Kwon

Kyung Hee University

Hye-Jin Ahn

Kyung Hee University

Su Jin Jeong

Kyung Hee University Medical Center

Min Kyung Shin ( $\square$ haddal@hanmail.net)

Kyung Hee University

\section{Research Article}

Keywords: allergic contact dermatitis, allergic rhinitis, asthma, atopic dermatitis, keloid

Posted Date: July 13th, 2021

DOI: https://doi.org/10.21203/rs.3.rs-690756/v1

License: (c) (i) This work is licensed under a Creative Commons Attribution 4.0 International License. Read Full License 


\section{Abstract}

Atopic dermatitis (AD) is associated with allergic comorbidities, such as asthma, allergic rhinitis (AR), and allergic contact dermatitis (ACD). The etiology of keloid is largely unknown; however, AD and keloid share inflammatory pathways characterized by T-helper cell 2 cytokines and increased dermal fibroblast activity. The prevalence of keloids is known to increase in patients with AD. This study aimed to estimate the prevalence of keloids in patients with $A D$, and compare it with the prevalence of other comorbidities of AD. We assessed the Korean National Health Information Database and medical records including coexisting asthma, AR, and ACD. Single and multiple logistic regression models were created for keloids and each allergic disease. The prevalence of keloids was higher in the AD group than in the control group. Among patients with $A D$, adolescents and adults had a higher prevalence of keloids than infants and children. The risk of keloids was high with $A D$ alone, and coexisting asthma significantly increased the risk. Similarly, the risk of keloids was higher in AR associated with AD and ACD associated with AD than in $A D$ alone. Thus, among Koreans, patients with $A D$ have a higher risk of keloid development, with coexisting allergic diseases increasing the risk.

\section{Introduction}

Atopic dermatitis $(A D)$ is a chronic inflammatory skin disease associated with numerous atopic and nonatopic comorbidities [1]. The prevalence of $A D$ is estimated to be $15-20 \%$ in children and $1-3 \%$ in adults, and the incidence has increased two- to three-fold during the past few decades in industrialized countries [2].

Keloid is a fibroproliferative dermal tumor resulting from skin trauma and subsequent abnormal scar formation [3]. The mechanism underlying the pathological development of keloids is largely unknown; however, it is mainly characterized by an overproduction of extracellular matrix and high activity of fibroblasts during the inflammatory and remodeling phases of scar formation, resulting in the excessive accumulation of collagen $[4,5]$. These processes are also observed in fibroblasts among patients with AD [6]. Although the association between AD and keloids is still unclear, several possible links have been hypothesized.

AD and keloid share common inflammatory pathways that are characterized by T-helper cell 2 (Th2) cytokines. An impaired inflammatory response of fibrogenesis involving Th2 cytokines secreted by CD $4+$ T cells such as interleukin (IL)-4, IL-5, IL-10, and IL-13, leads to the imbalance between pro-fibrotic and anti-fibrotic signals [7]. Additionally, in AD, upregulation of Th2 cytokines is triggered, and IL-4, IL-5, IL-10, and IL-13 are secreted, resulting in epidermal thickening, inflammation, pruritus, and decreased expression of barrier proteins such as filaggrin and loricrin, all of which are clinical characteristics of AD [8]. The reason why prurigo nodularis is frequently observed in patients with $A D$, with lichenification being one of the most common clinical manifestations of $A D$, is presumed to be due to this process. 
It is well established that patients with $A D$ have a higher chance of developing allergic comorbidities such as asthma, allergic rhinitis (AR), and allergic contact dermatitis (ACD) [1]. Furthermore, the severity of AD was associated with a higher prevalence of comorbid allergic disorders, as well as increased severity of comorbidities. Based on these facts, the authors hypothesized that patients with both $A D$ and allergic comorbidities might have a higher prevalence of keloids.

The prevalence of keloids throughout life is approximately $10 \%$ in Africa [9]. The prevalence is much lower in Asia, with the annual keloid incidence rate being $0.15 \%$ among the general Taiwanese population [10]. In a large-scale retrospective cohort study in Taiwan, the probability of keloid development in patients with $A D$ is 3.19 times higher than in the healthy population [11]. However, to date, there are no known reports on the prevalence of keloids in $A D$ accompanied by allergic diseases.

To the best of our knowledge, this study first analyzed big data from the Korean National Health Information Database (NHID) and aimed to 1) estimate the prevalence of keloids in patients with AD and 2) compare the prevalence of keloids with those of other comorbidities of $A D$.

\section{Materials And Methods}

\section{Data sources}

The data used in this study were obtained from the Korean NHID, in which diseases are coded according to the International Classification of Diseases, Tenth Revision, Clinical Modification (ICD-10-CM). This database contain nationwide claims data, and is linked at an individual level with the Korean registration number that is assigned to all Korean residents at birth or immigration for practical purposes. This study was approved by the Institutional Review Board of Kyung Hee University (IRB number 2019-07-076) and a waiver of informed consent was approved from IRB due to the retrospective nature of the big datasets. All methods were performed in accordance with the relevant guidelines and regulations.

\section{Study population}

We assessed the Korean NHID from 2017 to 2018, and a survey of 2,517,401 subjects with AD and $3,132,283$ non-AD subjects matched for age and sex. Medical records were analyzed according to the ICD-10-CM codes.

\section{Inclusion criteria of patients and classification of data}

We assessed 2,517,401 patients with AD from 2017 to 2018. A control group of 3,132,283 non-AD patients was extracted from a group that was not diagnosed with AD. All subjects in both groups were classified into two subgroups, with or without keloids. Demographic information (age and sex) and comorbidities of allergic diseases identified by ICD-10-CM codes were also collected. The associated 
allergic diseases in this study included asthma (ICD-10 code J304), AR (ICD-10 code L209), and ACD (ICD-10 code L239). All patients and the control group were classified by age: infant and early childhood, 0-5 years; late childhood, 6-10 years; adolescence, 11-20 years; adulthood, 21-60 years; and the elderly, $>61$ years.

\section{Statistical analysis}

We analyzed the chi-square test and Odds Ratio (OR) to see if there are differences in the frequency of occurrence of AD group and control group by age and gender. In the age group, the OR of late childhood, adolescence, adulthood, elderly was compared with infancy and early childhood as a reference. Also, in the sex, the OR of female was compared as male as a reference. In addition, we analyzed the variable that affect the risk of keloids according to the presence or absence of $A D$, age, and gender through the logistic regression. Furthermore, we analyzed the OR of the risk of keloids between the AD patients without asthma (or allergic rhinitis, allergic contact dermatitis) and with asthma (or allergic rhinitis, allergic contact dermatitis), and was compared to the control group through the multiple logistic regression adjusted by age, gender. A $p$-value of $<0.05$ was considered statistically significant. All data processing and statistical analyses were performed using SAS Enterprise Guide 7.1 (SAS Institute Inc., Cary, NC).

\section{Results}

\section{Baseline characteristics of patients}

Baseline characteristics of patients in the AD and control group are shown in Table 1. All personnel in this study were Koreans. A total of 5,649,684 personnel were included in this study, and $44.56 \%(2,517,401)$ had AD (see Supplementary Table. S1 online). In patients with AD, $26.01 \%$ of patients were younger than 5 years, and $51.88 \%$ were younger than 20 years of age. The rate of $A D$ tended to decrease with age. Of the patients with $A D, 53.01 \%$ were females. Among all age groups, the prevalence of $A D$ was higher only among infants and children but not among the remaining age groups.

Table 1

Baseline characteristics of patients with $A D$ and control group 


\begin{tabular}{|c|c|c|c|c|c|}
\hline Variables & $\begin{array}{l}\text { Atopic } \\
\text { dermatitis } \\
(n=2,517,401)\end{array}$ & $\begin{array}{l}\text { Controls } \\
(n=3,132,283)\end{array}$ & OR & $95 \% \mathrm{Cl}$ & $\begin{array}{l}p- \\
\text { value }\end{array}$ \\
\hline \multicolumn{6}{|l|}{ Age group, $\mathrm{n}(\%)$} \\
\hline $\begin{array}{l}\text { Infancy and early } \\
\text { childhood }\end{array}$ & $654,796(48.29)$ & $701,299(51.71)$ & 1.000 & & \\
\hline Late childhood & 329,795 (45.34) & $397,638(54.66)$ & 0.888 & $\begin{array}{l}0.883- \\
0.893\end{array}$ & $<0.001$ \\
\hline Adolescence & $321,596(43.62)$ & $415,745(56.38)$ & 0.828 & $\begin{array}{l}0.824- \\
0.833\end{array}$ & $<0.001$ \\
\hline Adulthood & $877,329(42.70)$ & $\begin{array}{l}1,177,245 \\
(57.30)\end{array}$ & 0.798 & $\begin{array}{l}0.795- \\
0.802\end{array}$ & $<0.001$ \\
\hline Elderly & $333,885(43.12)$ & $440,356(56.88)$ & 0.812 & $\begin{array}{l}0.808- \\
0.817\end{array}$ & $<0.001$ \\
\hline \multicolumn{6}{|l|}{ Sex, n (\%) } \\
\hline Men & $\begin{array}{l}1,182,857 \\
(44.80)\end{array}$ & $\begin{array}{l}1,457,194 \\
(55.20)\end{array}$ & 1.000 & . & \\
\hline Women & $\begin{array}{l}1,334,544 \\
(44.34)\end{array}$ & $\begin{array}{l}1,675,089 \\
(55.66)\end{array}$ & 0.981 & $\begin{array}{l}0.978- \\
0.985\end{array}$ & $<0.001$ \\
\hline \multicolumn{6}{|c|}{$\begin{array}{l}\text { Baseline characteristics of patients in the AD and control group. All personnel in this study were } \\
\text { Koreans. }\end{array}$} \\
\hline \multicolumn{6}{|c|}{ The $p$-value was obtained through the chi-square test. $\mathrm{Cl}$; confidence interval } \\
\hline
\end{tabular}

\section{Increased prevalence of keloids in the AD group}

The prevalence of keloids was higher in the AD group than in the control group (Table 2). Keloids exist in $1.43 \%(36,090$ out of $2,517,401)$ of the patients with $A D$ and in $0.52 \%(16,216$ out of $3,132,283)$ of the control group. Simple logistic regression was performed, and the prevalence of keloids was significantly higher among the patients with AD than in the control group (1.43\% vs. $0.52 \%$, OR: $2.795,95 \%$ confidence interval [CI]: 2.743-2.847, $p<0.0001)$. When comparing the prevalence of keloids according to age groups of all patients, the prevalence of keloids was higher among adolescents and adulthood than those belonging to infancy and early childhood (OR: $18.333,95 \% \mathrm{Cl}: 17.755-18.930$ and OR: $29.289,95 \% \mathrm{Cl}$ : 28.399-30.208 each, $p<0.0001)$. The entire numbers can be found as Supplementary Table. S2 online.

Table 2

Prevalence of keloids in patients with and without $A D$ 


\begin{tabular}{llllll} 
& $\mathrm{N}$ & Keloid $(\%)$ & OR & $95 \% \mathrm{Cl}$ & $p$-value \\
\hline Control & $3,132,283$ & $16,216(0.52)$ & 1.000 &. &. \\
\hline AD & $2,517,401$ & $36,090(1.43)$ & 2.795 & $2.743-2.847$ & $<0.001$ \\
\hline Age group, $\mathrm{n}(\%)$ & & & & & \\
\hline Infancy and early childhood & $1,537,360$ & $4,108(0.27)$ & 1.000 &. &. \\
\hline Late childhood & 857,092 & $6,463(0.75)$ & 2.836 & $2.727-2.949$ & $<0.001$ \\
\hline Adolescence & 968,628 & $45,350(4.68)$ & 18.333 & $17.755-18.930$ & $<0.001$ \\
\hline Adulthood & $3,520,791$ & $256,188(7.28)$ & 29.289 & $28.399-30.208$ & $<0.001$ \\
\hline Elderly & $1,343,210$ & $37,627(2.80)$ & 10.757 & $10.415-11.110$ & $<0.001$ \\
\hline Sex, $\mathrm{n}(\%)$ & & & & & \\
\hline Men & & & & & $<$ \\
\hline Women & $3,868,370$ & $147,039(3.80)$ & 1.000 &. & $<0.001$ \\
\hline
\end{tabular}

The effect of presence or absence of $A D$ on the risk of keloids was evaluated using the simple logistic regression.

$\mathrm{AD}$, atopic dermatitis; $\mathrm{Cl}$, confidence interval

\section{The effect of allergic comorbidities associated with AD on keloids}

The effect of having allergic comorbidities associated with $A D$ on the risk of developing keloids (Table 3 ) was evaluated using multiple logistic regression. Patients with $A D$ alone were at a higher risk of keloids (OR: 2.97; 95\% Cl: 2.91-3.03, $p<0.001$ ), and asthma coexisting with $A D$ increased the risk of keloids significantly (OR 3.17; $95 \% \mathrm{Cl} 3.08-3.26, p<0.001)$. This effect was also observed in AR and ACD. In patients with $A R$ associated with $A D$, the risk of keloids increased compared with that in $A D$ alone (OR 3.2; $95 \% \mathrm{Cl} 3.13-3.26$ vs. OR $2.61 ; 95 \% \mathrm{Cl} 2.54-2.67, p<0.001)$. In patients with $\mathrm{ACD}$ associated with $\mathrm{AD}$, the risk of keloids increased compared with that in $\mathrm{AD}$ alone (OR 3.81; $95 \% \mathrm{Cl} 3.74-3.89$ vs. OR 2.32; $95 \% \mathrm{Cl}$ 2.27-2.38, $p<0.001)$. The entire numbers can be found as Supplementary Table. S2 online.

Table 3

OR of keloids according to the type of allergic diseases associated with $A D$ 


\begin{tabular}{llllll} 
Variable & Classification & OR & $95 \% \mathrm{Cl}$ & \multicolumn{2}{c}{$p$-value } \\
\hline Asthma & Control & 1.000 & & & \\
\cline { 2 - 6 } & AD without asthma & 2.968 & 2.911 & 3.026 & $<0.001$ \\
\cline { 2 - 6 } & AD with asthma & 3.171 & 3.080 & 3.264 & $<0.001$ \\
\hline AR & Control & 1.000 & & & \\
\cline { 2 - 6 } & AD without AR & 2.606 & 2.542 & 2.673 & $<0.001$ \\
\hline ACD with AR & Control & 3.196 & 3.133 & 3.259 & $<0.001$ \\
\cline { 2 - 7 } & AD without ACD & 1.000 & & & $<0.001$ \\
\cline { 2 - 6 } & AD with ACD & 2.324 & 2.273 & 2.376 & $<0.001$
\end{tabular}

The effect of having allergic comorbidities associated with $A D$ on the risk of keloids was evaluated using multiple logistic regression adjusted for age and sex.

$A D$, atopic dermatitis; $A R$, allergic rhinitis; $A C D$, allergic contact dermatitis; $\mathrm{Cl}$ : confidence interval

\section{Discussion}

This is the first study demonstrating that the risk of keloids is strongly associated with AD in the Korean population. Our results also demonstrate that the risk of keloids increases with coexisting allergic diseases, such as asthma, AR, and ACD. Previous data on the prevalence of keloids is based mainly on Caucasians and Taiwanese in Asia [10,11]. In Korea, where all citizens are obligated to subscribe to national medical insurance, big data research using electronic medical information is feasible. For the first time, our findings show that the prevalence of keloids increased in patients with AD in the Korean population. Hajdarbegovic et al. also suggested that keloids may be strongly associated with atopic asthma in European, African, and Asian populations [12]. The findings in this study are consistent with those of previous studies wherein the risk of keloids is higher in AD patients with allergic comorbidities.

The molecular pathophysiology of keloid formation and progression is poorly understood. However, fibroblasts, one of the main cells responsible for most of the collagen and extracellular matrix deposition in both normal and abnormal wound healing, have been identified to contribute to keloid formation and dermal structural changes in AD skin lesions [13]. Recently, Shin et al. reported that keloidal fibroblasts treated with thymic stromal lymphopoietin (TSLP) produced significantly increased collagen I, collagen III, and transforming growth factor $\beta$ [14]. TSLP, an IL-7-like cytokine, is thought to induce upregulation of Th2-inflammatory cytokines, and there is increased expression of the TSLP gene in AD skin lesions [15]. 
Another molecule that contributes to the collagen production of dermal fibroblasts in AD is Oncostatin M (OSM), a T lymphocyte/macrophage-derived proinflammatory signaling molecule similar to the IL-6 cytokine family [16]. Fibroblasts are target cells for OSM. OSM stimulates collagen and glycosaminoglycan production in dermal fibroblasts, and this process can be observed in both keloidal formation and prurigo nodularis in patients with AD [17].

Other than upregulation of Th2 cytokines, effector memory CD8 + T cells and CD103 + CD8 + resident memory T (TRM) cells are increased in keloid tissue. Therefore, increased CD8 + TRM in keloid tissues might contribute to local inflammation [18]. TRM cells have been shown to contribute to the recurrence of AD; however, the exact roles of skin TRM are still unclear [19].

There have been a few reports on the association between allergic diseases and keloid development; however, these results are still debated. Hajdarbegovic et al., for example, revealed no association between $A D$ and keloids in the adjusted model of a case-control study. Additionally, they suggested that asthma was significantly, consistently, and strongly associated with keloids. However, there were no consistent associations found on keloids with atopic eczema or hay fever [12]. In contrast, Lu et al. demonstrated that patients with $A D$ had a greater possibility of developing keloids in a nationwide retrospective cohort study in Taiwan. According to this study, the AD cohort had significantly higher percentages of patients with asthma ( 25.41 vs. $12.91, p<0.001)$, AR ( 54.78 vs. $35.92, p<0.001)$, and allergic conjunctivitis (57.22 vs. $42.08, p<0.001)$ compared with the non-AD cohort [11]. In our study, AD patients with ACD also had an increased prevalence of keloid risk, which is different from previous studies, suggesting that ACD may have the characteristic of AD-associated comorbidity. In the past, the higher permeation of contact allergens through the disrupted skin barrier was the main factor of ACD in patients with $A D$. Recently, potential shared immune pathways have been demonstrated for subsets of $A D$ and ACD, including Th1, Th2, Th9, and/or Th17 [20, 21].

Han et al. demonstrated the relationship between a disintegrin and metalloprotease 33 (ADAM33) polymorphism and keloid scars in an East Asian population. Q-1 SNPs in blood were significantly associated with keloid scars. ADAM33 protein is a zinc-dependent endopeptidase, characterized by a prodomain, metalloprotease domain, and disintegrin domain [22]. The ADAM33 gene was found to be associated with asthma and airway hyperresponsiveness [23]. It is thought that this may explain the increased keloid risk in $A D$ associated with asthma. Considering the literature mentioned above, $A D$ and keloid share various genetic and pathologic mechanisms.

One of the strengths of this study is the use of a large population-based claims dataset consisting of one ethnic group, which enabled the analysis of all cases of $A D$, keloids, and comorbid allergic diseases. Moreover, since the Korean NHID is one of the largest claims datasets including all age groups and the entire region, the possibility of selection bias is reduced to compare the relationships between the $A D$ patients and control group. Second, it is the first time that big data analysis has demonstrated the result of an increase in the prevalence of keloids in AD patients with allergic diseases. However, this study has several limitations. First, because the analyzed medical records were originally collected for national 
insurance purposes, not for research purposes, additional demographic or social histories were unavailable. Second, due to the drawbacks of big data analysis regarding control variables such as age and sex, we used multiple logistic regression models to match those of the two groups. This systematic problem may result in confounding factors.

In conclusion, this study demonstrated that in the Korean population, patients with $A D$ have a higher risk of keloid development, and the risk is even higher with coexisting allergic diseases. Nevertheless, further exploration of this association is needed to confirm these relationships and the pathophysiological mechanisms common to $A D$ and keloids.

\section{Declarations}

\section{Availability of data and material}

All databases are available as Supplementary Information files.

\section{Acknowledgment}

We would like to thank Kang, D.,H. for supporting this work.

\section{Authors' contributions}

All authors contributed to the study conception and design. Conceptualization, funding acquisition, and supervision were performed by Shin, M., K. and Ahn, H., J. Data collection and analysis were performed by Jeong, S., J. and Kwon, H., E. The first draft of the manuscript was written by Kwon, H., E. and all authors commented on previous versions of the manuscript. All authors read and approved the final manuscript.

\section{Funding}

This work was supported by a grant from the Kyung Hee University in 2018 (KHU-20181041).

\section{Conflicts of interest}

The authors declare that they have no conflict of interest.

\section{Ethical approval}


This article does not contain any studies with human participants or animals performed by any of the authors. This research study was conducted retrospectively from data obtained for clinical purposes. An IRB of Kyung hee university hospital officially granted ethical approval.

\section{Consent to participate/Consent to publish}

Not applicable

\section{References}

1. Silverberg, J. I. Comorbidities and the impact of atopic dermatitis. Ann. Allergy. Asthma. Immunol.123, 144 - 51(2019).

2. Nutten, S. Atopic dermatitis: global epidemiology and risk factors. Ann. Nutr. Metab, 66 (Suppl 1), 816 (2015).

3. Bran, G. M., Goessler, U. R., Hormann, K., Riedel, F. \& Sadick, H. Keloids: current concepts of pathogenesis (review). Int. J. Mol. Med, 24, 283-293 (2009).

4. Fujiwara, M., Muragaki, Y. \& Ooshima, A. Keloid-derived fibroblasts show increased secretion of factors involved in collagen turnover and depend on matrix metalloproteinase for migration. Br. J. Dermatol, 153, 295-300 (2005).

5. Nakaoka, H., Miyauchi, S. \& Miki, Y. Proliferating activity of dermal fibroblasts in keloids and hypertrophic scars. Acta. Derm. Venereol, 75, 102-104 (1995).

6. Berroth, A. et al. Role of fibroblasts in the pathogenesis of atopic dermatitis. J. Allergy. Clin. Immunol, 131, 1547-1554 (2013).

7. Tripathi, S. et al. Hypertrophic scars and keloids: a review and current treatment modalities.biomed. Dermatol.4 (2020).

8. Brandt, E. B. \& Sivaprasad, U. Th2 Cytokines and Atopic Dermatitis. J. Clin. Cell. Immunol, 2, 110 (2011).

9. Mari, W. et al. Novel Insights on Understanding of Keloid Scar: Article Review. J. Am. Coll. Clin. Wound. Spec, 7, 1-7 (2016).

10. Sun, L. M., Wang, K. H. \& Lee, Y. C. Keloid incidence in Asian people and its comorbidity with other fibrosis-related diseases: a nationwide population-based study. Arch. Dermatol. Res, 306, 803-808 (2014).

11. Lu, Y. Y. et al. Keloid risk in patients with atopic dermatitis: a nationwide retrospective cohort study in Taiwan. BMJ Open, 8, e022865 (2018).

12. Hajdarbegovic, E. \& Bloem, A. The Association between Atopic Disorders and Keloids: A Case-control Study. Indian. J. Dermatol, 60, 635 (2015).

13. Andrews, J. P. et al. The paradigm of skin fibrosis - Pathomechanisms and treatment. Matrix Biol, 51, 37-46 (2016). 
14. Shin, J. U. et al. TSLP Is a Potential Initiator of Collagen Synthesis and an Activator of CXCR4/SDF-1 Axis in Keloid Pathogenesis. J. Invest. Dermatol, 136, 507-515 (2016).

15. Lee, E. B. et al. Increased serum thymic stromal lymphopoietin in children with atopic dermatitis. Pediatr. Allergy. Immunol, 21, e457-60 (2010).

16. Williams, K. A., Huang, A. H., Belzberg, M. \& Kwatra, S. G. Prurigo nodularis: Pathogenesis and management. J. Am. Acad. Dermatol, 83, 1567-1575 (2020).

17. Duncan, M. R., Hasan, A., Berman, B. \& Oncostatin M stimulates collagen and glycosaminoglycan production by cultured normal dermal fibroblasts: insensitivity of sclerodermal and keloidal fibroblasts. J. Invest. Dermatol, 104, 128-133 (1995).

18. Chen, Z. et al. Characterization of CD45RO + memory T lymphocytes in keloid disease. Br. J. Dermatol, 178, 940-950 (2018).

19. Xu, Q. N. \& Zhu, H. Q. The Roles of Skin-Resident Memory T Cells in Dermatoses. Int. J. Dermatol. Venereol, 2, 29-32 (2019).

20. Owen, J. L., Vakharia, P. P. \& Silverberg, J. I. The Role and Diagnosis of Allergic Contact Dermatitis in Patients with Atopic Dermatitis. Am. J. Clin. Dermatol, 19, 293-302 (2018).

21. Thyssen, J. P., McFadden, J. P. \& Kimber, I. The multiple factors affecting the association between atopic dermatitis and contact sensitization. Allergy, 69, 28-36 (2014).

22. Han, J. et al. Association of ADAM33 gene polymorphisms with keloid scars in a northeastern Chinese population. Cell. Physiol. Biochem, 34, 981-987 (2014).

23. Van Eerdewegh, P. et al. Association of the ADAM33 gene with asthma and bronchial hyperresponsiveness. Nature, 418, 426-430 (2002).

\section{Supplementary Files}

This is a list of supplementary files associated with this preprint. Click to download.

- Supplementarymaterialfinal.xlsx 